Service social

\title{
Un amour de père, par le collectif Cœur-Atout, Montréal, Saint-Martin, 1987, 238 pages.
}

\section{Jules-H. Gourgues}

Volume 37, numéro 1-2, 1988

Par-delà les barrières des sexes

URI : https://id.erudit.org/iderudit/706400ar

DOI : https://doi.org/10.7202/706400ar

Aller au sommaire du numéro

Éditeur(s)

École de service social de l'Université Laval

ISSN

1708-1734 (numérique)

Découvrir la revue

Citer ce compte rendu

Gourgues, J.-H. (1988). Compte rendu de [Un amour de père, par le collectif Cœur-Atout, Montréal, Saint-Martin, 1987, 238 pages.] Service social, 37(1-2),

275-277. https://doi.org/10.7202/706400ar d'utilisation que vous pouvez consulter en ligne.

https://apropos.erudit.org/fr/usagers/politique-dutilisation/ 
absents, passifs ou "aidants", peuvent gagner la garde, non parce que les mères sont inadéquates ou que les pères sont des parents vraiment égaux, mais parce qu'ils sont des hommes. 》 (p. 394).

Un ouvrage à lire absolument par les intervenantes et intervenants, les juges, etc. Le Québec n'est sûrement pas épargné, mon expérience personnelle m'ayant déjà mis en contact avec une scientifique à qui on avait retiré la garde de ses enfants parce qu'elle travaillait!

Francine LAVOIE

École de psychologie,

Université Laval.

Un amour de père, par le collectif Cœur-Atout, Montréal, Saint-Martin, 1987, 238 pages.

Un amour de livre que cet Amour de père!

Mais situons d'abord les auteurs et le contexte. Le collectif Cœur-Atout est formé surtout d'ex-membres du groupe qui a été à la barre du bateau, un peu intrépide pour l'époque, qui avait pour nom Hom-Info. Revue sur la condition masculine publiée à Montréal durant plus de cinq ans, elle a dû tristement disparaître en 1985, faute d'argent. Les couleurs et du groupe et du livre s'en trouvent alors toutes révélées. Résolument consacré à la condition masculine, le collectif a organisé en juin 1986 sa première grande activité, le colloque national sur L'intervention auprès des hommes. ${ }^{1}$ Fort de ce succès, il répéta l'expérience en juin 1987 avec le colloque sur La part du père. Un amour de père en constitue les actes. Le livre présente un bon aperçu sur l'état de la condition paternelle d'ici. Réalité troublée et troublante, s'il en est, la paternité s'y trouve soumise avec soin au stéthoscope psychosocial, sans panique, mais sans complaisance. Même si ce n'est pas ici le lieu de les présenter toutes, le livre fourmille d'idées pas forcément neuves, mais nouvellement formulées souvent.

D'abord celles développées par Jean Monbourquette. Signataire de deux chapitres, il présente quelques réflexions dignes d'arrêt. Il affirme notamment le rôle essentiel des groupes d'hommes dans la démarche nécessaire de " masculinisation " des hommes : " ces groupes d'hommes [...] forment un nouvel utérus qui engendre le jeune homme à une masculinité pleine et plus affermie " (p. 156). Pour paraître abusive et complaisante, la position se comprend mieux quand on l'inscrit dans le contexte psychanalytique où la situe l'auteur. Une question alors : comment s'explique l'évolution de tortue qui caractérise partout les groupes d'hommes s'il faut y voir quelque chose d'aussi essentiel ? N'est-ce pas, par contre, réussir à mettre en mots cette espèce de climat, d'esprit, très particulier que reconnaît généralement à ces groupes tout participant qui s'y est engagé à fond ? À propos de l'évolution sociale qu'on a vécue, dans la foulée du féminisme, sur le plan des rapports entre les sexes, il prétend qu'elle s'est 
malheureusement trop embourbée dans des discussions réductrices, obnubilées à l'excès par les seules dénonciations du sexisme. Qu'on ose affronter de la sorte les nouvelles censures ne représente-t-il pas un signe de bonne santé mentale pour ce mouvement par ailleurs fort positif ?

L'auteur se demande si la paternité, en tant que "présomption sociale », ne doit pas toujours comporter un acte d'adoption conscient et volontaire de la part du père tant biologique qu'autrement. Pour lui, le rôle du père en est un d'agent de "dé-fusion » de l'enfant à sa mère pour lui « créer une aire de liberté favorable à son autonomie " (p. 153). Enfin, Monbourquette voit dans le nombre record des suicides chez les jeunes et dans l'ampleur de la violence conjugale masculine des symptômes de "l'immense malaise" de la masculinité/paternité d'ici.

Un autre auteur très attendu parce qu'il est l'un de nos écrivains les plus prolifiques sur la condition masculine, mais aussi parce qu'il a vécu le mouvement de l'intérieur en tant qu'ex-membre du premier groupe d'hommes de Québec en 1980, c'est Marc Chabot. II prétend que, si on en est venu à savoir assez bien ce qu'on ne veut plus comme père, on ne sait pas encore pour autant avec clarté le père qu'on veut. Le "pseudo-père-mère " ne saurait être une rechange valable. "Avant le féminisme, le père n'avait des problèmes qu'avec le fils; depuis le féminisme, le père a maintenant des problèmes avec la mère et la fille aussi" (p. 27). De quel côté se tourner alors, pour ce paure père ? D'autant qu'on lui demande en outre de ne plus fuir dans le travail comme exutoire et de réintégrer le foyer au plus tôt. Un vrai cul-de-sac qui laisse songeur : peut-on construire solide sur du seul négatif et de la culpabilité seule? Particulière à l'auteur, ce dernier soulève la question du "père des enfants des autres", i.e. le père non biologique. Dommage qu'il ne lui fasse pas tellement dépasser le niveau de la préoccupation ; peut-être est-ce déjà une réponse en soi ? Mais, pour être très dur, son commentaire n'en est pas moins suave: "les hommes s'imaginent toujours qu'il suffit d'un coup de queue pour devenir père" (p. 29). Enfin, Chabot semble s'associer à la critique que formule aussi Monbourquette, à l'effet que le rôle paternel défini comme celui de faire accéder l'enfant au monde extérieur a fait son temps. Pour lui, la vraie solution du père de demain consistera à faire de la famille le vrai territoire du père. Révolutionnaire, en effet!

Arrive alors le chapitre qui se présente, et de loin, comme le plus en harmonie avec ce qu'il avance. Comment qualifier, en effet, toute cette belle brochette d'auteur-e-s qui dénoncent sans trop de gêne la sempiternelle disette émotionnelle du père, mais qui ne savent pas situer clairement leur propre discours sur le terrain qui s'impose ? Jacques Broué réussit à contourner le piège, et de façon tellement rafraîchissante. D'abord la question centrale dans l'aventure devenue périlleuse de la paternité : que se passe-t-il donc quand le père tente d'occuper auprès de l'enfant toute la place de coauteur et de co-éducateur qui lui revient? Pour Broué, son vécu de jeune père lui a fait subir jusqu'à la nausée des effets encore très réels du stéréotype social du père second violon. Au-delà de toute "théorisation", avec le meilleur des soucis de conformité au modèle très mode des "nouveaux pères ", c'est avec ses tripes que l'auteur témoigne des tristesses quotidiennes du vécu du jeune père, des blessures assez profondes 
associées aux accusations de "jalousie envers la mère». De là au "ressenti » concentré dans le seul registre de la honte et de la culpabilité - pourtant si contraire à la saine créativité - il n'y a qu'un pas : " la relation mère-enfant telle qu'investie dans notre société enferme la mère et l'enfant dans une dynamique d'exclusion des autres, d'exclusivité " (p. 75).

Une des bonnes trouvailles du livre, le coup de vérité de Broué trouve écho presque coup sur coup dans le chapitre d'Ann Pâquet-Deehy. Elle développe la version maternelle en posant la question : "les mères ont-elles une place à faire au père?" Confirmant d'abord les craintes que les mères ont d'être tenues encore une fois responsables du peu de place des pères, elle paraît bien réaliste de croire qu'on risque de se trouver devant une mère qui aide le père à prendre sa place. Pour elle, la vraie question est la capacité d'autonomie des pères: on croit lire qu'il n'y aurait pas de "problème de père" si les hommes savaient prendre leur place, non seulement comme père, mais aussi face au partage des rôles et des tâches domestiques. Pour l'auteure, une vraie " relation coopérative ne peut exister qu'entre des personnes égales" (p. 95) : c'est là essentiellement que se trouve le problème. Sans cette fichue égalité acquise une fois pour toutes, il y aura toujours un risque énorme que "le soin des enfants devienne le champ de bataille où se résolvent les autres conflits du couple" (ibid). Réponse pertinente au questionnement sous-entendu de Broué sur les capacités réelles du couple de survivre à l'enfant...

Enfin, il y aurait bien d'autres idées du livre à commenter :

- celles soulevées par André Masse autour du questionnement existentiel du père face à l'embarrassant sujet de la reproduction et de ses nouvelles technologies: "la grossesse chez l'autre demeure le seul moyen d'avoir accès à la parentalité" (p. 81) ;

- celles aussi que relève Claire Chamberland à qui revient d'avoir peint la fresque historique de la paternité au Québec (pp. 13-25) ;

- celles enfin de Willy Apollon dont la déclaration relative au "père pure invention sociale", dans les reportages de presse sur le colloque, avait causé tout un remous. ${ }^{2}$

Bref, Un amour de père est certainement un livre dont il vaut la peine de faire la lecture et de poursuivre la recherche, autant pour les intervenant-e-s auprès des pères que pour le commun des hommes et des femmes concerné-e-s par la paternité et l'égalité parentale.

Jules-H. GOURGUES

Ministère de la santé et des services sociaux, Québec.

\section{Références}

1 Collectif Cœur-Atout, Intervention auprès des hommes, Montréal, Fédération des C.L.S.C. du Québec, 1986, 117p. 